Review

\title{
Economic Considerations in Using HCV and HIV Positive Donors for Kidney Transplant
}

Aniruddha Bhattacharyya ${ }^{1,}{ }^{*}$, Lee R. Friedman ${ }^{2}$, Beje S. Thomas ${ }^{3,+, *}$, Coleman I. Smith ${ }^{4,+, *}$

1. Department of Medicine, University of Maryland Medical Center Midtown Campus, 827 Linden Ave, Baltimore, MD, USA; E-Mail: aniruddha.bhattacharyya@umm.edu

2. Georgetown University School of Medicine, 3900 Reservoir Road NW, Washington, DC, USA; EMail: Irf44@georgetown.edu

3. Department of Transplant Nephrology, Medstar Georgetown Transplant Institute, 3800 Reservoir Road NW, Washington, DC, USA; E-Mail: Beje.Thomas@gunet.georgetown.edu

4. Department of Transplant Hepatology, MedStar Georgetown Transplant Institute, 3800 Reservoir Road NW, Washington, DC, USA; E-Mail: Coleman.I.Smith@gunet.georgetown.edu

+ Co-senior author

* Correspondences: Aniruddha Bhattacharyya, Beje S. Thomas and Coleman I. Smith; E-Mails: aniruddha.bhattacharyya@umm.edu; Beje.Thomas@gunet.georgetown.edu; Coleman.I.Smith@gunet.georgetown.edu

Academic Editor: Haval Shirwan

Special Issue: Utilization of HCV and HIV Positive Donors

OBM Transplantation

2021, volume 5, issue 4

doi:10.21926/obm.transplant.2104154
Received: August 29, 2021

Accepted: October 11, 2021

Published: October 18, 2021

\begin{abstract}
End Stage Renal Disease is becoming more prevalent in the United States of America, with demand for kidney transplant exceeding the available organ supply. A novel method to increase the donor pool has been to consider transplanting organs from deceased patients who have had Hepatitis C Virus (HCV) or Human Immunodeficiency Virus (HIV) infections. Transplants with HCV infected kidneys are becoming more prevalent, due to increased organ supply due to increased mortality from injection opioid use. Similarly, deceased donor
\end{abstract}

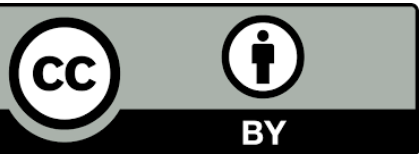

(C) 2021 by the author. This is an open access article distributed under the conditions of the Creative Commons by Attribution License, which permits unrestricted use, distribution, and reproduction in any medium or format, provided the original work is correctly cited. 
transplants using kidneys infected with HIV have become more common following the passage of the "HIV Organ Policy Equity (HOPE) Act" in 2013. These novel transplant strategies present distinct socioeconomic impacts which differ from those of prior transplant practices. Here, we have reviewed the costs and benefits of receiving a kidney transplant from deceased donors infected with HIV or HCV, compared to receiving a non-viremic kidney transplant.

\section{Keywords}

HIV; HCV; kidney; transplant; economics; opioid; quality; cost-effectiveness

\section{Introduction}

In the United States of America (USA), the incidence of End Stage Renal Disease (ESRD) has been progressively increasing, and with it the utilization of in-center hemodialysis [1]. While dialysis serves as a bridge to kidney transplant, the demand for organs continues to outstrip the available supply [2]. The waitlist for kidney transplantation has exceeded 100000 patients [3], with only $9.1 \%$ of these patients receiving a kidney transplant after 5 years [4]. A novel method to increase the donor pool has been to consider transplanting organs from deceased patients who have had Hepatitis C Virus (HCV) or Human Immunodeficiency Virus (HIV) infections [5, 6].

In 2013, the United States Congress passed the "HIV Organ Policy Equity (HOPE) Act", allowing organs from donors with HIV to be utilized for recipients with HIV [6]. As treatments for HIV have improved, patients have increasingly survived into older age. Along with this decreased mortality, the frequency of chronic diseases such as chronic kidney disease (CKD) has also increased [7]. Through the HOPE Act, older patients with HIV have been afforded greater consideration for receiving a transplant. Though the HOPE Act increases the organ donor pool available to patients with ESRD and HIV, there are additional costs and barriers that affect transplantation rates within this cohort.

Over the last few decades, there has also been an increasing incidence of HCV, associated with the rise of injection opioid use [8]. HCV has a 1-2\% prevalence among the American population and represents at least $4 \%$ of the potential organ donors [4]. Organ procurement from patients with $\mathrm{HCV}$ is projected to increase due to deaths caused by the opioid epidemic $[9,10]$. Currently, there are over 70000 deaths per year due to hypoxia from opioid overdoses [3, 5]. People who had HCV and died from opioid overdose are usually younger and healthier compared to other deceased donors $[9,11]$; though historically, nearly $40 \%$ of organs from donors with HCV have been discarded [3].

Transplants using donor organs from patients with HCV and HIV are becoming more common for many different organs; however, there is a paucity of data on the economic impacts of this novel approach. The most robust economic data for viremic organ transplant is available for kidney transplants. In this review article, we provide an overview of deceased donor kidney transplants (DDKT) using organs from donors with HIV and HCV, as well as describe the associated financial and societal costs from the patient and healthcare payer perspectives. 


\section{Transplants using Hepatitis C Organs}

In industrialized countries, HCV transmission occurs primarily through recreational injection drug use [12] such as heroin, or other opioids. Based on the current mortality rate of the opioid epidemic, HCV viremic organs are projected to represent $10-15 \%$ of the deceased donor organ pool in America [10]. Previously, fewer than half of healthcare providers felt that transplant of an organ from a donor with viremic HCV (HCV D+) into a disease-free recipient (R-) should be a part of the standard of care [4]. More recently, acceptance of this practice has been steadily increasing. While some centers have made the transplant strategy of implanting organs from donors with viremic HCV into disease free recipients (HCV D+/R-) the standard of care, the majority of HCV D+/R- transplants performed thus far have happened in experimental settings that are unequally distributed throughout America [4, 10, 13]. The Eastern United States procures the most HCV D+ organs, and performs the most HCV D+/R- transplants $[10,13]$, however, the availability of organs from opioid related mortality is especially pronounced in the Midwest United States [10]. Differing transplant practices and rates of acquiring organs with HCV can affect treatment options and net healthcare expenses for individual patients.

Though HCV D+ organs represent an increasing share of the supply, organs from those who are "increased risk donors" (IRD)-such as those with a history of intravenous (IV) drug use or HCV infection-have historically been underutilized $[3,9,14]$. HCV D+/R-transplants are not universally favored due to many health care providers' concern that HCV may not be cured in a transplant recipient [4]. Prior treatments for $\mathrm{HCV}$, including intravenous pegylated interferon and oral ribavirin, had long courses of therapy that were difficult to complete [15]. Side effects of these medications, such as influenza like symptoms and severe depression for interferon, and hemolytic anemia for ribavirin [16], exacerbated difficulty with regimen adherence. Interferon had limited outpatient use due to its intravenous formulation and was contraindicated post-transplant as it was thought to cause acute rejection [15]. More recently, oral direct acting anti-viral therapy (DAA) for HCV now easily cures between 90 to $100 \%$ of all patients with the disease [8,12,17-19], compared to $64 \%$ cure from combination therapy with interferon and ribivarin [16]. Side effects of DAA, including fatigue, gastrointestinal symptoms, and anemia, are better tolerated by patients compared to interferon and ribavirin regimens. Due to less severe side effects, oral formulations, and once daily dosing, patients are better able to adhere to DAA.

Though there is concern that the long term DAA cure rate cannot be extrapolated to the population receiving transplants, many experimental transplant regimens show minimal to zero risk of prolonged HCV infection in organ recipients $[3,8,9,12,20,21]$. For recipients who were infected prior to transplant $(\mathrm{R}+)$, multiple regimens of DAA showed sustained viral response (SVR) at 12 weeks post-transplant [22]. Disease free recipients in the THINKER trial by Goldberg et al. showed $100 \%$ SVR at 12 weeks post-transplant with a regimen of Elbasvir- Grazoprevir [17]. The EXPANDER trial by Durand et al. further demonstrated this DAA regimen to be effective infection prophylaxis, where no transplant recipients developed HCV viremia as detected on Nucleic Acid Testing (NAT) [18]. Multiple retrospective studies further corroborate the SVR rates observed in both THINKER and EXPANDER trials [23-25]. Based on this data, HCV D+/R- transplants appear feasible if DAA is started before or shortly after transplant. The risk of transmitting HCV via transplant is extremely low if DAA is readily started prior to the R- patient showing signs or symptoms of infection [5]; however, insurance coverage of DAA is a concern [11]. In many prior studies, the cost of DAA was 
not paid by insurers due to the experimental nature of DAA use in this population. It remains to be seen if DAA will be consistently reimbursed by insurance payers for the use of organ transplant.

Though HCV D+/R-transplant is not without risk, it provides benefits for the patient's health and quality of life. Deceased HCV viremic donors are more likely to be young, and have fewer comorbidities such as diabetes and hypertension [8, 10]. Outcomes for HCV D+/R- transplants are generally equivalent or superior to matched recipients of organs from donors without HCV infection or viremia (HCV D-), especially if donor organs are from patients who died by opioid overdose $[3,4$, 9]. Patients who are willing to accept an HCV D+ kidney are on the transplant waitlist for 0.7 to 1.56 years, compared to a median wait time of 4 years for a D- kidney [3]. Patients who receive HCV $\mathrm{D}+/ \mathrm{R}$ - transplants spend on average 1.7 years on dialysis, compared to 4.1 years for patients awaiting an aviremic organ (Figure 1) [9]. Though improving, the annual all-cause mortality rate for patients on hemodialysis is approximately $17 \%$ [1], and patients who receive a kidney transplant have significantly lower excess mortality compared to dialysis dependent patients [3]. Over $90 \%$ of transplant physicians are inclined to offer HCV D+/R- transplants if it reduces time spent on the waitlist by 4 years; $40 \%$ of these physicians feel this strategy is beneficial if it decreases time on the waitlist by only 1 year [4]. Patients are also inclined to accept HCV D+ organs if they are able to avoid or stop dialysis. They additionally value spending less time on the waitlist and subjectively feel a greater benefit from receiving an organ from a younger, healthier donor [26].

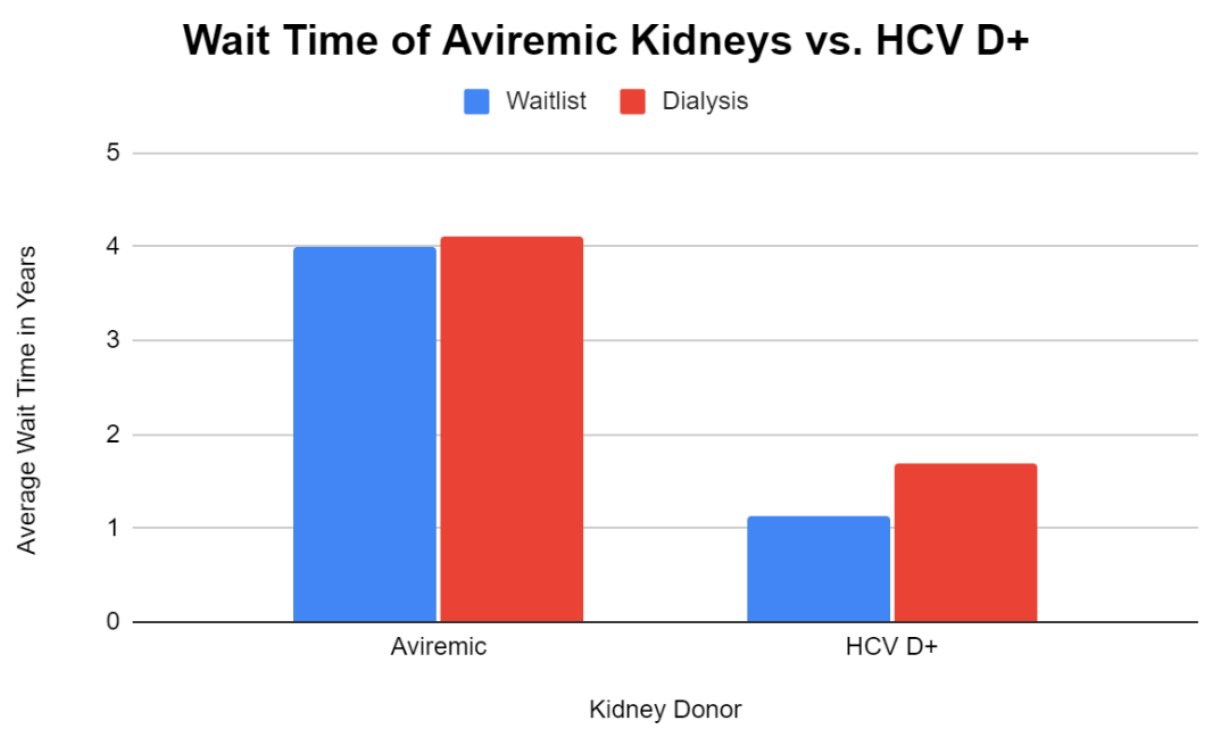

Figure 1 Bar graphs showing average time spent on wait list, and time spent requiring dialysis for patients receiving aviremic kidney transplant, compared to patients receiving transplants from donors with HCV. Average waiting time to receiving an organ with HCV is 1.12 years.

\section{Transplants using HIV Organs}

Due to advances in antiretroviral therapy (ART), HIV is now considered a "chronic disease rather than a fatal infection" [7]. As the mortality rate has decreased, the prevalence of ESRD in this population has increased [27]. The first kidney transplant given to a recipient with HIV (HIV R+) was performed in 2003 [28]. Though having HIV is no longer considered a contraindication to solid organ 
transplant, patients with HIV have been significantly less likely to be placed on the transplant waiting list, or receive any organ $[19,28]$. Prior to the HOPE Act, it was illegal to transplant organs from donors who had HIV (HIV D+). Currently, utilization of HIV D+ "organs remain limited to the research domain" [19].

Since the passage of the HOPE Act, 25 transplants using organs from donors with HIV to recipients with HIV (HIV D+/R+) have been performed through the "HOPE in Action Study" [6]. This is a multicenter open label pilot study comparing outcomes of this novel transplant strategy to conventional practice of implanting organs from donors without HIV (HIV D-) into recipients with HIV (HIV D-/R+). Among patients with HIV, there was no difference in 1 year mortality, graft function, or graft survival between the D+ and the D- cohorts [6]. HIV breakthrough was a rare occurrence in both groups and primarily occurred due to difficulty paying for ART. Patients with HIV who received HIV D+ organs spent 2 fewer years on dialysis compared to HIV D-/R+ patients [6]. Delayed graft function occurred more often in those who received HIV D- organs, which was attributed to greater incidence of HCV coinfection within that group. There was a greater incidence of graft rejection in HIV D+/R+ arm, though it failed to reach statistical significance.

Although recipients with HIV have higher rates of organ rejection [29], mortality and graft survival for these patients are comparable to transplant recipients without HIV [7]. Patients with HIV on dialysis have greater morbidity and mortality compared to dialysis patients without HIV [7, 30]. Data from the HOPE in Action study suggest that for patients with HIV and ESRD, accepting an organ from an HIV donor could decrease time on the waitlist and mitigate the need for dialysis. Using the HIV D+/R+ transplant strategy, the donor pool is projected to increase by up to 500 donors with HIV per year [19]. Due to the HOPE Act, organs that test falsely positive for HIV are much less likely to be discarded. Using organs from false positive donors may further "increase the donor pool by an estimated 50-100 donors per year" [29].

The main concern for using organs from donors with HIV is transmitting infection with a new or resistant strain to recipients $[7,29,30]$. There is also some concern for new HCV infection to recipients if donors have HIV and HCV coinfection [19]. To date, there has not been a kidney transplant from HIV donor given to a recipient without HIV (HIV D+/R-). However, there has been a case of HIV D+/R-pediatric liver transplant in South Africa [28]. These transplants pose new ethical challenges and may be considered depending on survivability without a transplant, size of donor pool, and efficacy of ART regimen. Though undetectable viral load likely makes HIV sexually untransmissible $(U=U)$, further research is required to validate this claim for patients with organ transplants [28].

\section{Current Economics of Transplantation}

Economic considerations of a medical intervention can be evaluated from multiple perspectives. For kidney transplants, monetary and non-monetary factors affect the assessment of cost effectiveness. To analyze this from the patient perspective, we can use lifetime cost savings from an intervention, as well as Quality Adjusted Life Years (QALY) gained. QALY represents the number of years lived weighted by an individual's preference for a certain level of health, aggregated over a population. QALY usually range from 0 to 1 where 0 represents death, and 1 represents living in perfect health for one additional year [31,32]. Outcomes that patients feel are "worse" than death are assigned negative values, while outcomes perceived to be better than a year of perfect health 
are scored over 1. For example, patients undergoing dialysis rate the baseline experience as 0.53 QALY. Following kidney transplant of a non-viremic organ, the average QALY improves to 0.84 (Figure 2) [12]. Thus, the intervention of transplant causes a gain of 0.31 QALY.

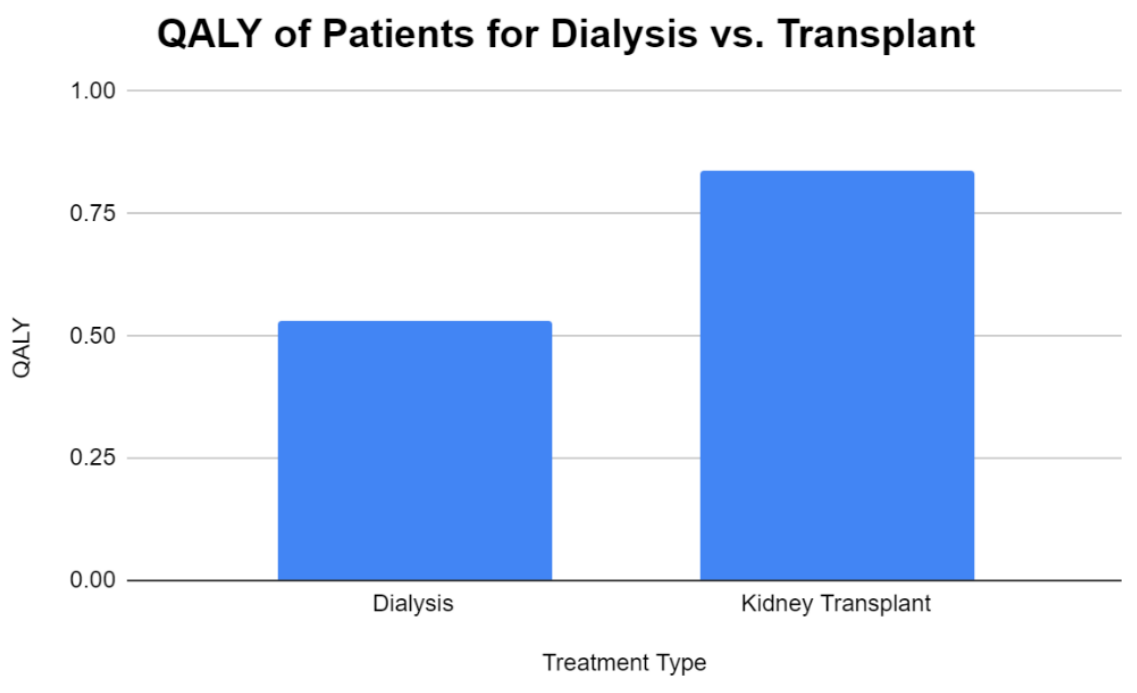

Figure 2 Bar graphs showing the respective QALY for patients on dialysis, compared to patients who received a kidney transplant. Receiving a kidney transplant causes a gain of 0.31 QALY compared to receiving dialysis.

From the perspective of a health care system, we can assess economic impact based on cost savings, cost effectiveness, and Disability Adjusted Life Years (DALY) prevented. DALY "reflects the sum of years of life lost due to premature mortality and years lived with disability" [31]. DALY also ranges from 0 to 1 , though 0 represents no disability and no premature mortality, while 1 represents the state of being dead. Unlike QALY which focuses on patient preference, DALY weighs definitions of disability based on an expert panel developed by the "Global Burden of Diseases, Injuries, and Risk Factors Initiative" (GBD) [31]. As DALY is based on standardized definitions of disability, this measure can be generalized across healthcare systems. Cost saving and cost effectiveness are also related but distinct measures. Cost saving interventions create a net decrease in the total healthcare dollars spent, while cost effective interventions cause a sufficiently large benefit relative to the price of intervention [33]. A cost effective intervention may or may not be cost saving.

A commonly used measure for cost effectiveness is the Incremental Cost-Effectiveness Ratio (ICER) - often expressed as dollars per QALY. ICER is a ratio evaluating the costs and benefits of one intervention compared to another [34]. It represents the average incremental cost in order to gain one additional QALY by using the intervention. ICER can be calculated "by dividing the difference in cost of 2 interventions by the difference of their effectiveness" [32]. ICER usually assesses cost effectiveness from the perspective of healthcare payers, such as Medicare in the USA [2, 35]. To determine whether an intervention is cost effective for the payer, the ICER is compared to a predetermined "cost effectiveness threshold". In the USA, this threshold is defined as $\$ 50000$ per QALY, benchmarked to the cost of treating ESRD under the criteria for Medicare enrollment in the 1970s [35]. For the purposes of this review, ICER would compare the utility of receiving a viremic kidney compared to the utility of not receiving a transplant and/or remaining on dialysis. 
The baseline costs of staying on dialysis or receiving a non-viremic kidney can be determined from Medicare expenditures. Treating ESRD accounts for $7.2 \%$ of total annual Medicare spending. The majority of this $\$ 36$ billion expense comes from hemodialysis [1]. As of 2017, the annual cost of providing hemodialysis was $\$ 91,795$ per person. In comparison, the annual cost to treat patients receiving a kidney transplant was $\$ 35,817$ (Figure 3) [1]. The rates of DDKT for patients on dialysis have decreased since 2000 and remained mostly stable between 2013-2017 [1]. As overall mortality rates for ESRD decrease, the total cost for dialysis is projected to increase.

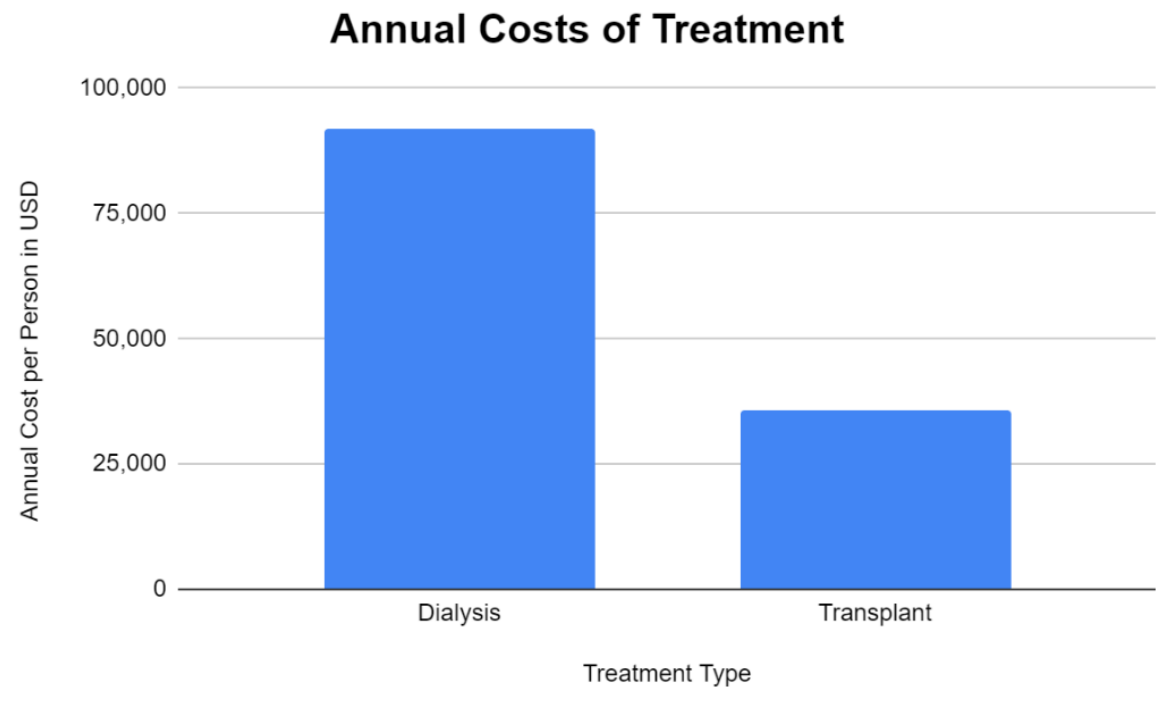

Figure 3 Bar graph comparing the annual cost of hemodialysis to the annual cost of kidney transplant per patient, measured in 2017 USD.

Cost forecasting for HCV D+/R- transplants has been performed with statistical modeling. The starting assumptions for these models were based on outcomes of previously discussed trials, and aggregate data from nonprofit organizations such as United Network of Organ Sharing (UNOS) and Scientific Registry of Transplant Recipients (SRTR). This forecasting was primarily done via Markov modeling [2, 3], and Monte Carlo (MC) simulations [12]. Markov models describe situations that involve stochastic sequential decision making overtime, where proportions of a starting group transition to a different state of health [32]. For example, one study iteratively stratifies patients that transition from requiring dialysis to a D-/R-group or to HCV D+/R-group that also receives DAA [3]. Another study models the annual proportion of a fixed cohort who transition from requiring dialysis to receiving an HCV D+/R- transplant [2]. Monte Carlo simulations generate bounds of uncertainty for variables whose outcomes are otherwise difficult to estimate or measure [32]. For instance, $\mathrm{MC}$ simulation could describe iterative transitions from remaining on dialysis to receiving a transplant if the probability of receiving a transplant is influenced by external factors. These models have been used to determine projected QALY, DALY, ICER, and cost savings. The MC simulation additionally provides insight into the limits of where these conclusions would continue to apply.

To holistically consider the economics of kidney transplantation, we must consider both patient specific measures - such as QALY, and system-based measures - such as DALY and ICER. QALY, and DALY both assess an intervention's impact on quality of life. DALY uses standardized definitions of 
disability whereas QALY aggregates subjective assessments determined by patient preference. ICER expresses the cost per QALY gained for an intervention in relation to the status quo therapy. Cost effectiveness is determined by comparing the ICER to a predetermined threshold. In the USA, this threshold is commonly set at $\$ 50000$ per QALY. All of the above measures are often calculated by extrapolating existing data via iterative modeling over time. Common techniques of iterative modeling include Markov modeling and MC modeling. For the purposes of this paper, all nominal monetary values have been converted to USD using data from Organisation for Economic Cooperation and Development (OECD) [36].

\section{Comparative Cost Outcomes for HCV Transplant}

Overall, receiving an HCV D+/R- transplant appears beneficial for both cost and quality of life. Compared to waiting for an aviremic organ, transplant with HCV infected kidneys improved QALY by 0.91 in the American population [3]. This was likely due to the much shorter wait time required to receive a viremic transplant. Markov modeling techniques from American data would suggest that receiving an HCV D+ transplant is associated with improved QALY as long as the average waiting time for a viremic kidney is less than 3.79 years [3]. The lifetime cost savings for a patient accepting a viremic kidney could approach $\$ 38000$ USD [3]. Based on MC simulations in Australia, an HCV $\mathrm{D}+/ \mathrm{R}$ - transplant strategy prevented an average of $0.15 \mathrm{DALY}$ when compared to remaining on the transplant waitlist for up to 5 years [12]. Additional Markov modeling using data from Canada suggests that receiving HCV D+ transplant increases QALY and is cost saving compared to staying on the waitlist for up to 5 years [2].

Transplant with HCV D+ kidneys remain cost saving if an organ is received up to 2.67 years on the waitlist [3]. It remains cost effective (ICER< $\$ 50000 / Q A L Y$ ) until waitlist time exceeds 3.1 years [3]. Compared to remaining on the transplant waitlist for one additional year, the ICER for receiving a HCV D+/R-transplant was $\$ 3578$ USD to gain 1 QALY $[2,36]$. When favoring a D+/R- strategy, the cumulative savings for healthcare payers over 5 years ranged from $\$ 25400-\$ 122400$ USD per patient depending on the duration of time spent awaiting a D- organ [2,36]. Considering all aspects of treatment after transplant- including “...DAA, immunosuppression, and provider care for up to 5 years," - the D+/R- transplant strategy saved approximately $\$ 191000$ USD per patient compared to the D-/R- cohort [37].

Pragmatically, the cost for DAA may be a significant barrier to developing $D+/ R$ - strategy as standard of care. For these transplant recipients, insurance providers may refuse to cover DAA due to lack of "medical necessity" [21]. Though this may hinder post-transplant treatment of HCV, it appears similar results can be achieved with a prophylactic course of DAA [21]. While the vast majority of patients are expected to achieve SVR with one course of DAA [17-19], an estimated 4\% may fail to respond to two courses of therapy [2]. For recipients who cannot clear HCV infection, the cost of ongoing DAA is comparable to the annual cost of dialysis, and the HCV D+/R-strategy is unlikely to be cost saving [12]. Cost effectiveness for transplant will vary by organ specific wait times (per country), HCV prevalence, country specific probability of death while on the wait list, and cost of HCV treatment. The annual probability of dying while waiting for a transplant was 14\% [12], though the mortality risk had increased in the setting of the COVID-19 pandemic [38]. Further studies are required to reassess total healthcare costs in the post pandemic setting. 


\section{Comparative Cost of HIV Treatment with and without Transplant}

The frequency of transplants using HIV D+ organs pales in comparison to HCV D+ transplants. Though the potential for greater utilization exists, there is currently not enough data to model costs for HIV D+/R+ transplants. However, subjective patient experiences and access to transplant can affect the downstream costs once HIV kidney transplants become more prevalent. The utilization of ART accounts for the vast majority of costs incurred by the average patient with HIV [39, 40]. Patients with ESRD and HIV must additionally bear over $\$ 90000$ USD per annum for hemodialysis $[1,3]$. Following transplant, continued insurance coverage of various ART regimens may also play a significant role in the total costs incurred by patients and healthcare systems alike.

America has the highest cost of ART worldwide, and this cost has steadily increased from 2012 to 2018, at 3.5 times the rate of expected inflation [41]. Even though generic ART options exist, the average annual price of a regimen can be over $\$ 36,000$ USD per patient [41]. Among completed HIV $\mathrm{D}+\mathrm{R}+$ transplants, lapses in ART due to loss of insurance coverage or unaffordable copay has occurred [6]. A large proportion of HIV patients are on Medicaid due to disabilities from the sequelae of HIV and are co-enrolled in Medicare based on the 2010 Patient Protection and Affordable Care Act. Though nearly $80 \%$ of Medicare patients with HIV qualify for coverage due to disability, an increasing proportion of patients receive Medicare due to being 65 years of age or older [42]. As patients continue to live longer, there is expected to be a greater incidence of ART related adverse events which can influence transplant related issues [39].

As patients with HIV live longer, there is expected to be a greater incidence and cost burden attributed to ART related ESRD [39]. Extrapolating this trend, it is increasingly likely that the pool of HIV patients needing kidney transplant will continue to increase $[6,7,29]$. HIV related mortality is decreasing far more rapidly than non-HIV related deaths [43], thus there is expected to be a shortage of HIV D+ organs relative to demand.

Patients with HIV who have ESRD face disease specific challenges for obtaining evaluation for kidney transplant [29]. In addition to meeting center specific requirements for kidney transplant, those with HIV must also have a CD4 count over 200, be on a stable ART regimen, and have an undetectable viral load $[19,29]$. Patients with HIV must also have regular follow up with physicians who routinely provide HIV oriented care [29]. Psychosocial stressors-such as psychiatric illness, unemployment, and alcohol and substance abuse- are more prevalent in patients with HIV [40]. These stressors may be an added barrier to meeting the criteria for transplantation [19, 30]. The additional psychosocial strain of deciding between remaining on the waiting list or taking an organ from a higher risk donor increases the difficulty of obtaining informed consent for transplant. The HOPE Act mandates that Independent Recipient Advocates (HIRA) must be involved to navigate these challenging decisions with patients [44]. HIRAs typically have backgrounds in nursing, social work, or medicine. Duties as the HIRA are often added on to primary clinical roles. In some instances, an individual HIRA has assisted with over 15 evaluations [44]. Thus far, HIRAs have been independent of the transplant team, and are unpaid volunteers. As HIV D+/R+ transplant becomes more frequent, the HIRA position may need to become a salaried role, or further compensation will likely be needed for clinical workers to take on this additional responsibility. 


\section{Conclusion}

Transplants using kidneys from deceased donors with HCV and HIV are viable solutions to addressing the current organ shortage. Due to increased mortality from the opioid epidemic, organs with HCV are projected to represent up to $15 \%$ of the available donor pool. The proportion of patients eligible for transplant are increasingly open to receiving an organ from a deceased donor with HCV. An overwhelming majority of HCV free patients who receive an HCV D+ kidney achieve SVR with DAA. Compared to remaining on the transplant waitlist or requiring hemodialysis, the HCV $D+/ R$ - transplant strategy is a cost-effective method for significantly improving quality of life. Despite the cost of DAA, healthcare payers such as Medicare can save over $\$ 190,000$ per patient over 5 years for transplant using HCV D+ kidneys compared to the usual HCV D-/R- approach. From the patient perspective, those who receive an HCV D+ kidney can save up to $\$ 38000$ USD over their lifetime compared to not receiving a transplant. HCV D+/R- transplants likely are cost saving and cost effective due to shorter waiting times for receiving a kidney.

HIV has become more of a chronic illness due to improvements in ART. An increasing share of patients with HIV have ESRD. The donor pool for this population is increased through the HOPE Act, which allows for transplants using organs from donors with HIV. There is insufficient data to extrapolate costs associated with HIV D+/R+ transplants. Insurer coverage of ART, and the need for additional staff such as HIRA to navigate increased psychosocial stressors experienced by those with HIV are likely to significantly impact the economics of this transplant strategy.

\section{Acknowledgments}

The authors would like to thank the University of Maryland Midtown Campus, Georgetown University School of Medicine, and Medstar Georgetown Transplant Institute for facilitating the collaborative environment needed to create this article.

\section{Author Contributions}

Aniruddha Bhattacharyya MD: was involved in the initial conception of the article, wrote the initial draft of the review article, and edited drafts for economic content. Lee R. Friedman MS: developed the figures for this submission, and copy edited drafts of this review article. Beje S. Thomas MD: is a co-senior author who was involved in the initial conception of the article, and edited drafts for clinical content. Coleman I. Smith MD FRACP FAASLD: is a co-senior author who proposed review article, was involved in the initial conception of the article, and edited drafts for clinical content.

\section{Funding}

There is no funding support for this review article.

\section{Competing Interests}

All authors declare that there are no competing interests. 


\section{References}

1. Saran R, Robinson B, Abbott KC, Agodoa LYC, Bragg-Gresham J, Balkrishnan R, et al. US renal data system 2018 annual data report: Epidemiology of kidney disease in the United States. Am J Kidney Dis. 2019; 73: A7-A8.

2. Kadatz M, Klarenbach S, Gill J, Gill JS. Cost-effectiveness of using kidneys from hepatitis C nucleic acid test-positive donors for transplantation in hepatitis C-negative recipients. Am J Transplant. 2018; 18: 2457-2464.

3. Eckman MH, Woodle ES, Thakar CV, Alloway RR, Sherman KE. Cost-effectiveness of using kidneys from HCV-viremic donors for transplantation into HCV-uninfected recipients. Am J Kidney Dis. 2020; 75: 857-867.

4. Couri T, Katz J, Stoeckle K, Nugooru A, Yeh H, Chung R, et al. Provider attitudes toward the use of hepatitis $C$ virus-positive organs in kidney transplantation. Am J Nephrol. 2019; 50: 168-176.

5. Dao A, Cuffy M, Kaiser TE, Loethen A, Cafardi J, Luckett K, et al. Use of HCV Ab+/NAT- donors in HCV naïve renal transplant recipients to expand the kidney donor pool. Clin Transplant. 2019; 33: e13598.

6. Durand CM, Zhang W, Brown DM, Yu S, Desai N, Redd AD, et al. A prospective multicenter pilot study of HIV-positive deceased donor to HIV-positive recipient kidney transplantation: HOPE in action. Am J Transplant. 2021; 21: 1754-1764.

7. Katou S, Vogel T, Morgul H, Eichelmann AK, Becker F, Slepecka P, et al. ABO-incompatible living donor kidney transplantation in a human immunodeficiency virus-positive recipient from a human immunodeficiency virus-positive donor: A case report. Transplant Proc. 2020; 52: 27392741.

8. Sadowski BW, Smith $\mathrm{Cl}$. Use of organs from hepatitis $\mathrm{c}$ viremic donors: Addressing the needs of a changing waitlist and the effect of a public health crisis. OBM Hepatol Gastroenterol. 2020; 4. Doi: 10.21926/obm.hg.2003049.

9. Bowring MG, Shaffer AA, Massie AB, Cameron A, Desai N, Sulkowski M, et al. Center-level trends in utilization of HCV-exposed donors for HCV-uninfected kidney and liver transplant recipients in the United States. Am J Transplant. 2019; 19: 2329-2341.

10. Ariyamuthu VK, Sandikci B, AbdulRahim N, Hwang C, MacConmara MP, Parasuraman R, et al. Trends in utilization of deceased donor kidneys based on hepatitis $C$ virus status and impact of public health service labeling on discard. Transpl Infect Dis. 2020; 22: e13204.

11. Burton Jr JR, Terrault NA, Goldberg DS, Bloom RD, Gilroy R, Heimbach JK, et al. Liver and kidney recipient selection of hepatitis $C$ virus viremic donors: Meeting consensus report from the 2019 controversies in transplantation. Transplantation. 2020; 104: 476-481.

12. Scott N, Snell G, Westall G, Pilcher D, Raggatt M, Walker RG, et al. Cost-effectiveness of transplanting lungs and kidneys from donors with potential hepatitis $C$ exposure or infection. Sci Rep. 2020; 10: 1459.

13. King KL, Husain SA, Mohan S. Trends in transplantation center use of kidneys from deceased donors with positive hepatitis $C$ virus nucleic acid testing. Am J Kidney Dis. 2020; 76: 743-746.

14. Sapiano MR, Jones JM, Bowman J, Levi ME, Basavaraju SV. Impact of us public health service increased risk deceased donor designation on organ utilization. Am J Transplant. 2019; 19: 2560-2569. 
15. Gordon CE, Francis J. Hepatitis C treatment in dialysis patients: Is a new dawn approaching? Am J Kidney Dis. 2014; 64: 178-180.

16. Liu CH, Huang CF, Liu CJ, Dai CY, Liang CC, Huang JF, et al. Pegylated interferon- $\alpha 2 a$ with or without low-dose ribavirin for treatment-naive patients with hepatitis $C$ virus genotype 1 receiving hemodialysis: A randomized trial. Ann Intern Med. 2013; 159: 729-738.

17. Goldberg DS, Abt PL, Reese PP, THINKER Trial Investigators. Transplanting HCV-infected kidneys into uninfected recipients. N Engl J Med. 2017; 377: 1104-1105.

18. Durand CM, Bowring MG, Brown DM, Chattergoon MA, Massaccesi G, Bair N, et al. Direct-acting antiviral prophylaxis in kidney transplantation from hepatitis $C$ virus-infected donors to noninfected recipients: An open-label nonrandomized trial. Ann Intern Med. 2018; 168: 533540.

19. Sawinski D. Kidney transplantation in patients with HIV. Kidney360. 2020; 1: 705-711.

20. Molnar MZ, Nair S, Cseprekal O, Yazawa M, Talwar M, Balaraman V, et al. Transplantation of kidneys from hepatitis C-infected donors to hepatitis C-negative recipients: Single center experience. Am J Transplant. 2019; 19: 3046-3057.

21. Gupta G, Yakubu I, Bhati CS, Zhang Y, Kang L, Patterson JA, et al. Ultra-short duration direct acting antiviral prophylaxis to prevent virus transmission from hepatitis $C$ viremic donors to hepatitis $C$ negative kidney transplant recipients. Am J Transplant. 2020; 20: 739-751.

22. Chen K, Lu P, Song R, Zhang J, Tao R, Wang Z, et al. Direct-acting antiviral agent efficacy and safety in renal transplant recipients with chronic hepatitis $C$ virus infection: A PRISMAcompliant study. Medicine. 2017; 96: e7568.

23. Kwok RM, Ahn J, Schiano TD, Te HS, Potosky DR, Tierney A, et al. Sofosbuvir plus ledispasvir for recurrent hepatitis $C$ in liver transplant recipients. Liver Transplant. 2016; 22: 1536-1543.

24. Morales AL, Liriano-Ward L, Tierney A, Sang M, Lalos A, Hassan M, et al. Ledipasvir/sofosbuvir is effective and well tolerated in postkidney transplant patients with chronic hepatitis $C$ virus. ClinTransplant. 2017; 31: e12941.

25. Nookala AU, Crismale J, Schiano T, Te H, Ahn J, Robertazzi S, et al. Direct-acting antiviral regimens are safe and effective in the treatment of hepatitis $C$ in simultaneous liver-kidney transplant recipients. Clin Transplant. 2018; 32: e13198.

26. Van Pilsum Rasmussen SE, Seaman S, Brown D, Desai N, Sulkowski M, Segev DL, et al. Patient's perspectives of experimental HCV-positive to HCV-negative renal transplantation: Report from a single site. AJOB Empir Bioeth. 2020; 11: 40-52.

27. Bickel M, Marben W, Betz C, Khaykin P, Stephan C, Gute P, et al. End-stage renal disease and dialysis in HIV-positive patients: Observations from a long-term cohort study with a follow-up of 22 years. HIV Med. 2013; 14: 127-135.

28. Botha J, Fabian J, Etheredge H, Conradie F, Tiemessen CT. HIV and solid organ transplantation: Where are we now. Curr HIV AIDS Rep. 2019; 16: 404-413.

29. Blumberg EA, Rogers CC. Solid organ transplantation in the HIV-infected patient: Guidelines from the American Society of Transplantation Infectious Diseases Community of Practice. Clin Transplant. 2019; 33: e13499.

30. Boyle SM, Fehr K, Deering C, Raza A, Harhay MN, Malat G, et al. Barriers to kidney transplant evaluation in HIV-positive patients with advanced kidney disease: A single-center study. Transplant Infect Dis. 2020; 22: e13253. 
31. Feng X, Kim DD, Cohen JT, Neumann PJ, Ollendorf DA. Using QALYs versus DALYs to measure cost-effectiveness: How much does it matter? Int J Technol Assess Health Care. 2020; 36: 96103.

32. Komorowski M, Raffa J. Markov models and cost effectiveness analysis: Applications in medical research. In: Secondary Analysis of Electronic Health Records. Cham: Springer International Publishing; 2016. pp.351-367.

33. Neumann P, Cohen J, Goodell S. Cost savings and cost-effectiveness of clinical preventive care. Synth Proj Res Synth Rep. 2009; 18: 48508.

34. Arnold M, Griffin S, Ochalek J, Revill P, Walker S. A one stop shop for cost-effectiveness evidence? Recommendations for improving disease control priorities. Cost Eff Resour Alloc. 2019; $17: 7$.

35. McDougall JA, Furnback WE, Wang BC, Mahlich J. Understanding the global measurement of willingness to pay in health. J Market Access Health Policy. 2020; 8: 1717030.

36. Organisation for Economic Co-operation and Development. Exchange rates [Internet]. Paris: Organisation for Economic Co-operation and Development; 2021 [cited 2021 May 8th]. Available from: https://data.oecd.org/conversion/exchangerates.htm?fbclid=IwAR2Bn6JIF8WfAbVhwDEiKFhGO rsTuiLq1PW47o1ujebwc7CWF0e0wUx A.

37. Gupta G, Zhang Y, Carroll NV, Sterling RK. Cost-effectiveness of hepatitis C-positive donor kidney transplantation for hepatitis C-negative recipients with concomitant direct-acting antiviral therapy. Am J Transplant. 2018; 18: 2496-2505.

38. Miller J, Wey A, Musgrove D, Ahn YS, Hart A, Kasiske BL, et al. Mortality among solid organ waitlist candidates during COVID-19 in the United States. Am J Transplant.2021; 21: 2262-2268.

39. Ward T, Sugrue D, Hayward O, McEwan P, Anderson S, Lopes S, et al. Estimating HIV management and comorbidity costs among aging HIV patients in the United States: A systematic review. J Manag Care Spec Pharm. 2020; 26: 104-116.

40. Cohen JP, Beaubrun A, Ding Y, Wade RL, Hines DM. Estimation of the incremental cumulative cost of HIV compared with a non-HIV population. Pharmacoeconomics Open. 2020; 4: 687-696.

41. McCann NC, Horn TH, Hyle EP, Walensky RP. HIV antiretroviral therapy costs in the United States, 2012-2018. JAMA Intern Med. 2020; 180: 601-603.

42. Tseng CW, Dudley RA, Chen R, Walensky RP. Medicare part D and cost-sharing for antiretroviral therapy and preexposure prophylaxis. JAMA Netw Open. 2020; 3: e202739.

43. Bosh KA, Johnson AS, Hernandez AL, Prejean J, Taylor J, Wingard R, et al. Vital signs: Deaths among persons with diagnosed HIV infection, United States, 2010-2018. Morb Mortal Wkly Rep. 2020; 69: 1717-1724.

44. Bollinger JM, Eno A, Seaman S, Brown D, Van Pilsum Rasmussen SE, Tobian AA, et al. Early experiences of independent advocates for potential HIV+ recipients of HIV+ donor organ transplants. Clin Transplant. 2019; 33: e13617. 


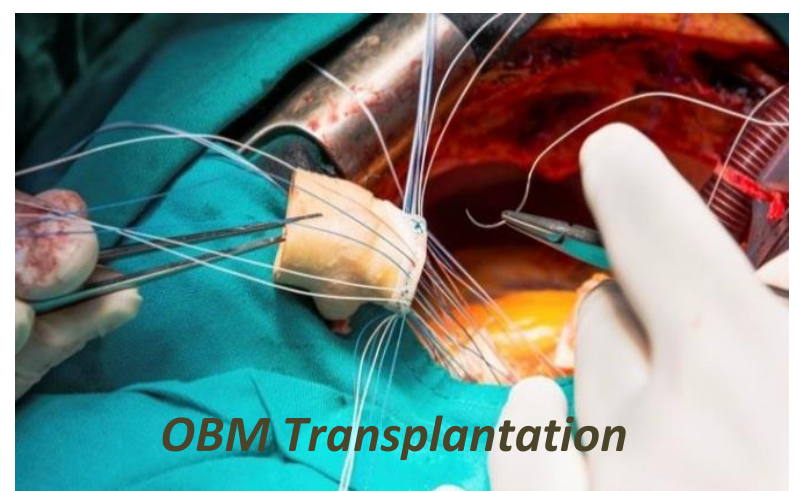

Enjoy OBM Transplantation by:

1. Submitting a manuscript

2. Joining in volunteer reviewer bank

3. Joining Editorial Board

4. Guest editing a special issue

For more details, please visit:

http://www.lidsen.com/journals/transplantation 